\title{
A new modified generalized Laguerre operational matrix of fractional integration for solving fractional differential equations on the half line
}

\author{
Ali H Bhrawy ${ }^{1,2^{*}}$, Mohammed M Alghamdi ${ }^{1}$ and Taha M Taha ${ }^{2}$
}

${ }^{*}$ Correspondence:

alibhrawy@yahoo.co.uk

1 Department of Mathematics,

Faculty of Science, King Abdulaziz

University, Jeddah, 21589, Saudi

Arabia

${ }^{2}$ Department of Mathematics,

Faculty of Science, Beni-Suef

University, Beni-Suef, Egypt

\begin{abstract}
In this paper, we derived a new operational matrix of fractional integration of arbitrary order for modified generalized Laguerre polynomials. The fractional integration is described in the Riemann-Liouville sense. This operational matrix is applied together with the modified generalized Laguerre tau method for solving general linear multi-term fractional differential equations (FDEs). Only small dimension of a modified generalized Laguerre operational matrix is needed to obtain a satisfactory result. Illustrative examples reveal that the present method is very effective and convenient for linear multi-term FDEs on a semi-infinite interval.
\end{abstract}

Keywords: operational matrix; modified generalized Laguerre polynomials; tau method; multi-term FDEs; Riemann-Liouville fractional integration

\section{Introduction}

Fractional differential equations have drawn the interest of many researchers (see, for instance, [1-4]) due to their important applications in science and engineering. Indeed, we can find numerous applications in viscoelasticity, electrochemistry, control, electromagnetic, porous media, and so forth. In consequence, the subject of fractional differential equations is gaining much importance and attention. For some recent developments on the subject, see [5-13] and the references therein.

Spectral methods employ orthogonal systems as the basis functions and so usually provide accurate numerical results [14-17]. The usual spectral methods are only available for bounded domains for solving FDEs; see [6, 18-20]. However, many problems in science, engineering, and finance are set on unbounded domains. Therefore, it is also interesting to consider spectral tau methods for solving multi-term FDEs on the half line by using the operational matrix of fractional integration of modified Laguerre polynomials.

Some authors developed the Laguerre spectral method for the half line for ordinary, partial, and delay differential equations; see [21-25]. A new family of generalized Laguerre polynomials is introduced in [21], and in [24] Yan and Guo proposed a collocation method for solving initial value problems of second-order ODEs by using modified Laguerre functions.

(c) 2012 Bhrawy et al: licensee Springer. This is an Open Access article distributed under the terms of the Creative Commons Attribution License (http://creativecommons.org/licenses/by/2.0), which permits unrestricted use, distribution, and reproduction in any medium, provided the original work is properly cited. 
In the last few years, there has been a growing interest in the use of spectral method for numerical treatments of FDEs in bounded domains. Saadatmandi and Dehghan [18] proposed an operational Legendre tau technique for the numerical solution of multi-term FDEs. The same technique based on the operational matrix of Chebyshev polynomials has been used for the same problem (see [26]). In [27], Doha et al. derived the Jacobi operational matrix of fractional derivatives which was applied together with the spectral tau method for a numerical solution of general linear multi-term fractional differential equations. Bhrawy et al. [20] used a quadrature shifted Legendre tau method for treating multi-term linear FDEs with variable coefficients. Moreover, in [28] the authors developed a Jacobi-Gauss-Lobatto collocation method for solving the nonlinear fractional Langevin equation with three-point boundary conditions.

Recently, El-Danaf et al. [29] investigated the use of spline functions of an integral form to approximate the solution of fractional order differential equations. In [30], Kadem and Baleanu introduced the Chebyshev spectral approach for a fractional radiative transfer equation, in which the multidimensional problem has been transformed into a system of fractional differential equations. Also, the waveform relaxation method in solving fractional differential equations is applied in [31]. A new explicit formula for the integrals of shifted Chebyshev polynomials of any degree for any fractional order in terms of shifted Chebyshev polynomials themselves is derived in [32], and a fast algorithm is developed for the solution of linear multi-order fractional differential equations by considering their integrated forms. Moreover, a new operational Jacobi matrix of fractional integration is investigated in [33] for solving linear FDEs on a bounded domain.

The operational matrix of integer integration has been determined for several types of orthogonal polynomials such as Chebyshev polynomials [34] and Laguerre and Hermite polynomials[35]. Recently, Singh et al. [36] derived the Bernstein operational matrix of integration. More recently, Bhrawy and Alofi [37] proposed the operational Chebyshev matrix of fractional integration in the Riemann-Liouville sense which was applied together with the spectral tau method to solve linear FDEs on a finite interval.

Up to now, and to the best of our knowledge, most of formulae corresponding to those mentioned previously are unknown and are traceless in the literature for fractional integration in the Riemann-Liouville sense. This partially motivates our interest in the operational matrix of fractional integration for modified generalized Laguerre polynomials.

In this paper, we propose a new tau method based on the operational matrix of fractional integration in the Riemann-Liouville sense, in which we take the modified Laguerre functions as the basis functions and approximate the solutions of multi-term FDEs on a semi-infinite interval. Finally, the accuracy of the proposed algorithm is demonstrated by test problems.

The remainder of the paper is organized as follows. The next section is for preliminaries. In Section 3, we derive the modified generalized Laguerre operational matrix of fractional integration. In Section 4, we apply the modified generalized Laguerre operational matrix of fractional integration to solve linear multi-order FDEs. In Section 5, the proposed method is applied to two examples. Also, a conclusion is given in Section 6. 


\section{Some basic preliminaries}

The most used definition of fractional integration is due to Riemann-Liouville, which is as follows:

$$
J^{v} f(x)=\frac{1}{\Gamma(v)} \int_{0}^{x}(x-t)^{\nu-1} f(t) d t, \quad v>0, x>0, \quad \text { and } \quad J^{0} f(x)=f(x) .
$$

One of the basic properties of the operator $J^{v}$ is

$$
J^{v} x^{\beta}=\frac{\Gamma(\beta+1)}{\Gamma(\beta+1+\nu)} x^{\beta+\nu} .
$$

The next equation defines Riemann-Liouville fractional derivative of order $v$

$$
D^{v} f(x)=\frac{d^{m}}{d x^{m}}\left(J^{m-v} f(x)\right)
$$

where $m-1<v \leq m, m \in N$, and $m$ is the smallest integer greater than $v$.

$$
\text { If } m-1<v \leq m, m \in N \text {, then }
$$

$$
D^{v} J^{v} f(x)=f(x), \quad J^{v} D^{v} f(x)=f(x)-\sum_{i=0}^{m-1} f^{(i)}\left(0^{+}\right) \frac{x^{i}}{i !}, \quad x>0 .
$$

Now, let $\Lambda=(0, \infty)$ and $w^{(\alpha, \beta)}(x)=x^{\alpha} e^{-\beta x}$ be a weight function on $\Lambda$ in the usual sense. Define

$$
L_{w^{(\alpha, \beta)}}^{2}(\Lambda)=\left\{v \mid v \text { is measurable on } \Lambda \text { and }\|v\|_{w^{(\alpha, \beta)}}<\infty\right\} \text {, }
$$

equipped with the following inner product and norm:

$$
(u, v)_{w^{(\alpha, \beta)}}=\int_{\Lambda} u(x) v(x) w^{(\alpha, \beta)}(x) d x, \quad\|v\|_{w^{(\alpha, \beta)}}=(u, v)_{w^{(\alpha, \beta)}}^{\frac{1}{2}} .
$$

Next, let $w^{(\alpha, \beta)}(x)$ with $\alpha>-1$ and $\beta>0$. The modified generalized Laguerre polynomial of degree $i$ is defined by

$$
L_{i}^{(\alpha, \beta)}(x)=\frac{1}{i !} x^{-\alpha} e^{\beta x} \partial_{x}^{i}\left(x^{i+\alpha} e^{-\beta x}\right), \quad i=0,1, \ldots
$$

According to (2.2)-(2.4) of [21] for $\alpha>-1$ and $\beta>0$, we have

$$
\begin{aligned}
& L_{i}^{(\alpha, \beta)}(0)=\frac{\Gamma(i+\alpha+1)}{\Gamma(\alpha+1) i !}, \\
& \partial_{x} L_{i}^{(\alpha, \beta)}(x)=-\beta L_{i-1}^{(\alpha+1, \beta)}(x), \\
& L_{i+1}^{(\alpha, \beta)}(x)=\frac{1}{i+1}\left[(2 i+\alpha+1-\beta x) L_{i}^{(\alpha, \beta)}(x)-(i+\alpha) L_{i-1}^{(\alpha, \beta)}(x)\right], \quad i=1,2, \ldots,
\end{aligned}
$$

where $L_{0}^{(\alpha, \beta)}(x)=1$ and $L_{1}^{(\alpha, \beta)}(x)=-\beta x+\frac{\Gamma(\alpha+2)}{\Gamma(\alpha+1)}$. 
The set of modified generalized Laguerre polynomials is the $L_{w^{(\alpha, \beta)}}^{2}(0, \infty)$-orthogonal system, namely

$$
\int_{0}^{\infty} L_{j}^{(\alpha, \beta)}(x) L_{k}^{(\alpha, \beta)}(x) w^{(\alpha, \beta)}(x) d x=h_{k} \delta_{j k}
$$

where $\delta_{j k}$ is the Kronecker function and $h_{k}=\frac{\Gamma(k+\alpha+1)}{\beta^{\alpha+1} k !}$. The modified generalized Laguerre polynomial on the interval $\Lambda \equiv(0, \infty)$ is given by

$$
L_{i}^{(\alpha, \beta)}(x)=\sum_{k=0}^{i}(-1)^{k} \frac{\Gamma(i+\alpha+1) \beta^{k}}{\Gamma(k+\alpha+1)(i-k) ! k !} x^{k}, \quad i=0,1, \ldots
$$

The special value

$$
D^{q} L_{i}^{(\alpha, \beta)}(0)=\frac{(-1)^{q} \beta^{q} \Gamma(i+\alpha+1)}{(i-q) ! \Gamma(q+\alpha+1)}, \quad i \geq q
$$

will be of important use later.

\section{Modified generalized Laguerre operational matrix of fractional integration}

The main objective of this section is to derive an operational matrix of fractional integration for modified generalized Laguerre polynomials.

Let $u(x) \in L_{w^{(\alpha, \beta)}}^{2}(\Lambda)$, then $u(x)$ may be expressed in terms of modified generalized Laguerre polynomials as

$$
u(x)=\sum_{j=0}^{\infty} a_{j} L_{j}^{(\alpha, \beta)}(x), \quad a_{j}=\frac{1}{h_{k}} \int_{0}^{\infty} u(x) L_{j}^{(\alpha, \beta)}(x) w^{(\alpha, \beta)}(x) d x, \quad j=0,1, \ldots
$$

In practice, only the first $(N+1)$-terms modified generalized Laguerre polynomials are considered. Then we have

$$
u_{N}(x)=\sum_{j=0}^{N} a_{j} L_{j}^{(\alpha, \beta)}(x)=C^{T} \phi(x)
$$

where the modified generalized Laguerre coefficient vector $C$ and the modified generalized Laguerre vector $\phi(x)$ are given by

$$
C^{T}=\left[c_{0}, c_{1}, \ldots, c_{N}\right], \quad \phi(x)=\left[L_{0}^{(\alpha, \beta)}(x), L_{1}^{(\alpha, \beta)}(x), \ldots, L_{N}^{(\alpha, \beta)}(x)\right]^{T}
$$

Theorem 3.1 Let $\phi(x)$ be the modified generalized Laguerre vector and $v>0$, then

$$
J^{v} \phi(x) \simeq \mathbf{P}^{(v)} \phi(x)
$$


where $\mathbf{P}^{(v)}$ is the $(N+1) \times(N+1)$ operational matrix of fractional integration of order $v$ in the Riemann-Liouville sense and is defined as follows:

$$
\mathbf{P}^{(v)}=\left(\begin{array}{ccccc}
\Psi_{v}(0,0) & \Psi_{v}(0,1) & \Psi_{v}(0,2) & \ldots & \Psi_{\nu}(0, N) \\
\Psi_{v}(1,0) & \Psi_{v}(1,1) & \Psi_{\nu}(1,2) & \ldots & \Psi_{\nu}(1, N) \\
\vdots & \vdots & \vdots & \ldots & \vdots \\
\Psi_{\nu}(i, 0) & \Psi_{\nu}(i, 1) & \Psi_{v}(i, 2) & \ldots & \Psi_{v}(i, N) \\
\vdots & \vdots & \vdots & \ldots & \vdots \\
\Psi_{v}(N, 0) & \Psi_{v}(N, 1) & \Psi_{v}(N, 2) & \ldots & \Psi_{v}(N, N)
\end{array}\right),
$$

where

$$
\Psi_{v}(i, j)=\sum_{k=0}^{i} \sum_{r=0}^{j} \frac{(-1)^{k+r} \beta^{(-v)} j ! \Gamma(i+\alpha+1) \Gamma(k+v+\alpha+r+1)}{(i-k) !(j-r) ! r ! \Gamma(k+v+1) \Gamma(k+\alpha+1) \Gamma(\alpha+r+1)} .
$$

Proof Using the analytic form of the modified generalized Laguerre polynomials $L_{i}^{(\alpha, \beta)}(x)$ of degree $i(6)$ and (2), then

$$
\begin{aligned}
J^{v} L_{i}^{(\alpha, \beta)}(x)= & \sum_{k=0}^{i}(-1)^{k} \frac{\beta^{k} \Gamma(i+\alpha+1)}{(i-k) ! k ! \Gamma(k+\alpha+1)} J^{v} x^{k} \\
= & \sum_{k=0}^{i}(-1)^{k} \frac{\beta^{k} \Gamma(i+\alpha+1)}{(i-k) ! \Gamma(k+v+1) \Gamma(k+\alpha+1)} x^{k+v}, \\
& i=0,1, \ldots, N .
\end{aligned}
$$

Now, approximating $x^{k+v}$ by $N+1$ terms of modified generalized Laguerre series, we have

$$
x^{k+v}=\sum_{j=0}^{N} c_{j} L_{j}^{(\alpha, \beta)}(x),
$$

where $c_{j}$ is given from (8) with $u(x)=x^{k+v}$, that is,

$$
c_{j}=\sum_{r=0}^{j}(-1)^{r} \frac{\beta^{-k-v} j ! \Gamma(k+v+\alpha+r+1)}{(j-r) ! r ! \Gamma(r+\alpha+1)}, \quad j=1,2, \ldots, N .
$$

In virtue of (13) and (14), we get

$$
J^{v} L_{i}^{(\alpha, \beta)}(x)=\sum_{j=0}^{N} \Psi_{v}(i, j) L_{j}^{(\alpha, \beta)}(x), \quad i=0,1, \ldots, N,
$$

where

$$
\begin{aligned}
\Psi_{\nu}(i, j)= & \sum_{k=0}^{i} \sum_{r=0}^{j} \frac{(-1)^{k+r} \beta^{(-v)} j ! \Gamma(i+\alpha+1) \Gamma(k+v+\alpha+r+1)}{(i-k) !(j-r) ! r ! \Gamma(k+v+1) \Gamma(k+\alpha+1) \Gamma(\alpha+r+1)}, \\
& j=1,2, \ldots, N .
\end{aligned}
$$


Accordingly, Eq. (16) can be written in a vector form as follows:

$$
J^{v} L_{i}^{(\alpha, \beta)}(x) \simeq\left[\Psi_{v}(i, 0), \Psi_{v}(i, 1), \Psi_{v}(i, 2), \ldots, \Psi_{v}(i, N)\right] \phi(x), \quad i=0,1, \ldots, N
$$

Eq. (17) leads to the desired result.

Corollary 3.2 If we define the $q$ times repeated integration of the modified generalized Laguerre vector $\phi(x)$ by $J^{q} \phi(x)$, and

$$
J^{q} \phi(x) \simeq \mathbf{I}^{(q)} \phi(x)
$$

then $\mathbf{I}^{(q)}=\mathbf{P}^{(q)}$ is the operational matrix of integration of $\phi(x)$, where $q$ is an integer value.

\section{Modified generalized Laguerre tau method based on operational matrix}

Many practical problems are governed by initial value problems of multi-term FDEs. We may decompose such problems to a system of fractional equations of varying orders and then solve them numerically (see [38]). Whereas, for saving work, it seems reasonable to solve them directly.

In this section, the modified generalized Laguerre tau method based on the operational matrix is proposed to numerically solve the FDEs. The basic idea of this technique is as follows: (i) The FDE is converted to a fully integrated form via fractional integration in the Riemann-Liouville sense. (ii) Subsequently, the integrated form equations are approximated by representing them as linear combinations of modified generalized Laguerre polynomials. (iii) Finally, the integrated form equation is converted to an algebraic equation by introducing the operational matrix of fractional integration of the modified generalized Laguerre polynomials.

In order to show the fundamental importance of the modified generalized Laguerre operational matrix of fractional integration, we apply it to solve the following multi-order FDE:

$$
D^{v} u(x)=\sum_{i=1}^{k} \gamma_{j} D^{\beta_{i}} u(x)+\gamma_{k+1} u(x)+f(x), \quad \text { in } \Lambda=(0, \infty)
$$

with initial conditions

$$
u^{(i)}(0)=d_{i}, \quad i=0, \ldots, m-1,
$$

where $\gamma_{i}(i=1, \ldots, k+1)$ are real constant coefficients, $m-1<v \leq m, 0<\beta_{1}<\beta_{2}<\cdots<$ $\beta_{k}<v$, and $g(x)$ is a given source function. If we apply the Riemann-Liouville integral of order $v$ to (19) after making use of (4), we get the integrated form of (19), namely

$$
\begin{aligned}
& u(x)-\sum_{j=0}^{m-1} u^{(j)}\left(0^{+}\right) \frac{x^{j}}{j !}=\sum_{i=1}^{k} \gamma_{i} J^{v-\beta_{i}}\left[u(x)-\sum_{j=0}^{m_{i}-1} u^{(j)}\left(0^{+}\right) \frac{x^{j}}{j !}\right]+\gamma_{k+1} J^{v} u(x)+J^{v} f(x), \\
& u^{(i)}(0)=d_{i}, \quad i=0, \ldots, m-1,
\end{aligned}
$$


where $m_{i}-1<\beta_{i} \leq m_{i}, m_{i} \in N$. This implies that

$$
\begin{aligned}
& u(x)=\sum_{i=1}^{k} \gamma_{i} J^{\nu-\beta_{i}} u(x)+\gamma_{k+1} J^{v} u(x)+g(x), \\
& u^{(i)}(0)=d_{i}, \quad i=0, \ldots, m-1,
\end{aligned}
$$

where

$$
g(x)=J^{\nu} f(x)+\sum_{j=0}^{m-1} d_{j} \frac{x^{j}}{j !}+\sum_{i=1}^{k} \gamma_{i} j^{\nu-\beta_{i}}\left(\sum_{j=0}^{m_{i}-1} d_{j} \frac{x^{j}}{j !}\right) .
$$

In order to use the tau method based on the operational matrix of fractional integration for modified generalized Laguerre for solving the fully integrated problem (22) with initial conditions (20), we approximate $u(x)$ and $g(x)$ by the modified generalized Laguerre polynomials as

$$
\begin{aligned}
& u_{N}(x) \simeq \sum_{i=0}^{N} c_{i} L_{i}^{(\alpha, \beta)}(x)=C^{T} \phi(x), \\
& g(x) \simeq \sum_{i=0}^{N} g_{i} L_{i}^{(\alpha, \beta)}(x)=G^{T} \phi(x),
\end{aligned}
$$

where the vector $G=\left[g_{0}, \ldots, g_{N}\right]^{T}$ is given but $C=\left[c_{0}, \ldots, c_{N}\right]^{T}$ is an unknown vector.

Now, the Riemann-Liouville integral of orders $v$ and $\left(v-\beta_{j}\right)$ of the approximate solution (23), after making use of Theorem 3.1 (relation (11)), can be written as

$$
J^{v} u_{N}(x) \simeq C^{T} J^{v} \phi(x) \simeq C^{T} \mathbf{P}^{(v)} \phi(x)
$$

and

$$
J^{\nu-\beta_{j}} u_{N}(x) \simeq C^{T} J^{\nu-\beta_{j}} \phi(x) \simeq C^{T} \mathbf{P}^{\left(\nu-\beta_{j}\right)} \phi(x), \quad j=1, \ldots, k,
$$

respectively, where $\mathbf{P}^{(v)}$ is the $(N+1) \times(N+1)$ operational matrix of fractional integration of order $v$. Employing Eqs. (23)-(26), the residual $R_{N}(x)$ for Eq. (22) can be written as

$$
R_{N}(x)=\left(C^{T}-C^{T} \sum_{j=1}^{k} \gamma_{j} \mathbf{P}^{\left(\nu-\beta_{j}\right)}-\gamma_{k+1} C^{T} \mathbf{P}^{(v)}-G^{T}\right) \phi(x) .
$$

As in a typical tau method, we generate $N-m+1$ linear algebraic equations by applying

$$
\left\langle R_{N}(x), L_{j}^{(\alpha, \beta)}(x)\right\rangle=\int_{0}^{\infty} R_{N}(x) w^{(\alpha, \beta)}(x) L_{j}^{(\alpha, \beta)}(x) d x=0, \quad j=0,1, \ldots, N-m .
$$

Also, by substituting Eqs. (8) and (23) in Eq. (20), we get

$$
u^{(i)}(0)=C^{T} \mathbf{D}^{(i)} \phi(0)=d_{i}, \quad i=0,1, \ldots, m-1 .
$$


Eqs. (28) and (29) generate $N-m+1$ and $m$ set of linear equations, respectively. These linear equations can be solved for unknown coefficients of the vector $C$. Consequently, $u_{N}(x)$ given in Eq. (23) can be calculated, which gives a solution of Eq. (19) with the initial conditions (20).

\section{Illustrative examples}

To illustrate the effectiveness of the proposed method in the present paper, some examples are carried out in this section. The results obtained by the present method reveal that it is very effective and convenient for linear FDEs on the half line.

Example 1 Consider the FDE

$$
D^{2} u(x)+D^{\frac{3}{2}} u(x)+u(x)=x^{2}+2+\frac{4 x^{\frac{1}{2}}}{\Gamma(0.5)}, \quad u(0)=0, u^{\prime}(0)=0, x \in \Lambda,
$$

whose exact solution is given by $u(x)=x^{2}$.

If we apply the technique described in Section 4 with $N=2$, then the approximate solution can be written as

$$
u_{N}(x)=\sum_{i=0}^{2} c_{i} L_{i}^{(\alpha, \beta)}(x)=C^{T} \phi(x)
$$

and

$$
\begin{aligned}
\mathbf{P}^{(2)} & =\left(\begin{array}{lll}
\Psi_{2}(0,0) & \Psi_{2}(0,1) & \Psi_{2}(0,2) \\
\Psi_{2}(1,0) & \Psi_{2}(1,1) & \Psi_{2}(1,2) \\
\Psi_{2}(2,0) & \Psi_{2}(2,1) & \Psi_{2}(2,2)
\end{array}\right), \\
\mathbf{P}^{\left(\frac{1}{2}\right)} & =\left(\begin{array}{lll}
\Psi_{\frac{1}{2}}(0,0) & \Psi_{\frac{1}{2}}(0,1) & \Psi_{\frac{1}{2}}(0,2) \\
\Psi_{\frac{1}{2}}(1,0) & \Psi_{\frac{1}{2}}(1,1) & \Psi_{\frac{1}{2}}(1,2) \\
\Psi_{\frac{1}{2}}(2,0) & \Psi_{\frac{1}{2}}(2,1) & \Psi_{\frac{1}{2}}(2,2)
\end{array}\right), \quad G=\left(\begin{array}{l}
g_{0} \\
g_{1} \\
g_{2}
\end{array}\right) .
\end{aligned}
$$

Using Eq. (28), we obtain

$$
\begin{gathered}
\left(\Psi_{\frac{1}{2}}(0,2)+\Psi_{2}(0,2)\right) c_{0}+\left(\Psi_{\frac{1}{2}}(1,2)+\Psi_{2}(1,2)\right) c_{1} \\
+\left(1+\Psi_{\frac{1}{2}}(2,2)+\Psi_{2}(2,2)\right) c_{2}+g_{2}=0 .
\end{gathered}
$$

Now, by applying Eq. (29), we have

$$
\begin{aligned}
& c_{0}+\frac{\Gamma(\alpha+2)}{\Gamma(\alpha+1)} c_{1}+\frac{\Gamma(\alpha+3)}{2 \Gamma(\alpha+1)} c_{2}=0, \\
& -\beta c_{1}-\frac{\beta \Gamma(\alpha+3)}{\Gamma(\alpha+2)} c_{2}=0 .
\end{aligned}
$$

Finally, by solving Eqs. (31)-(33), we have the three unknown coefficients with general $\alpha$ and $\beta$ as follows:

$$
c_{0}=\frac{\alpha^{2}+3 \alpha+2}{\beta^{2}}, \quad c_{1}=\frac{-2 \alpha-4}{\beta^{2}}, \quad c_{2}=\frac{2}{\beta^{2}} .
$$


Table 1 The values of $c_{0}, c_{1}$ and $c_{2}$ for various choices of $\alpha$ and $\beta$ for Example 1

\begin{tabular}{|c|c|c|c|c|c|c|c|c|c|}
\hline $\bar{\alpha}$ & $\beta$ & $c_{0}$ & $c_{1}$ & $\overline{c_{2}}$ & $\bar{\alpha}$ & $\beta$ & $c_{0}$ & $c_{1}$ & $\overline{c_{2}}$ \\
\hline 0 & 1 & 2 & -4 & 2 & 0 & 2 & $\frac{1}{2}$ & -1 & $\frac{1}{2}$ \\
\hline 1 & & 6 & -6 & 2 & 1 & & $\frac{3}{2}$ & $-\frac{3}{2}$ & $\frac{1}{2}$ \\
\hline 2 & & 12 & -8 & 2 & 2 & & 3 & -2 & $\frac{1}{2}$ \\
\hline 3 & & 20 & -10 & 2 & 3 & & 5 & $-\frac{5}{2}$ & $\frac{1}{2}$ \\
\hline 4 & & 30 & -12 & 2 & 4 & & $\frac{15}{2}$ & -3 & $\frac{1}{2}$ \\
\hline 0 & 3 & $\frac{2}{9}$ & $-\frac{4}{9}$ & $\frac{2}{9}$ & 0 & 4 & $\frac{1}{8}$ & $-\frac{1}{4}$ & $\frac{1}{8}$ \\
\hline 1 & & $\frac{2}{3}$ & $-\frac{2}{3}$ & $\frac{2}{9}$ & 1 & & $\frac{3}{8}$ & $-\frac{3}{8}$ & $\frac{1}{8}$ \\
\hline 2 & & $\frac{4}{3}$ & $-\frac{8}{9}$ & $\frac{2}{9}$ & 2 & & $\frac{3}{4}$ & $-\frac{1}{2}$ & $\frac{1}{8}$ \\
\hline 3 & & $\frac{20}{9}$ & $-\frac{10}{9}$ & $\frac{2}{9}$ & 3 & & $\frac{5}{4}$ & $-\frac{5}{8}$ & $\frac{1}{8}$ \\
\hline 4 & & $\frac{10}{3}$ & $-\frac{4}{3}$ & $\frac{2}{9}$ & 4 & & $\frac{15}{8}$ & $-\frac{3}{4}$ & $\frac{1}{8}$ \\
\hline
\end{tabular}

Thus, we can write

$$
u(x)=\left(c_{0}, c_{1}, c_{2}\right)\left(\begin{array}{l}
L_{0}^{(\alpha, \beta)}(x) \\
L_{1}^{(\alpha, \beta)}(x) \\
L_{2}^{(\alpha, \beta)}(x)
\end{array}\right)=x^{2}
$$

which is the exact solution.

The values of $c_{0}, c_{1}, c_{2}$ with various choices of $\alpha$ and $\beta$ are listed in Table 1.

Example 2 Consider the following fractional initial value problem:

$$
\begin{aligned}
& D^{\frac{5}{2}} u(x)+3 u(x)=3 x^{3}+\frac{6}{\Gamma(1.5)} x^{\frac{1}{2}}, \\
& u(0)=0, u^{\prime}(0)=0, u^{\prime \prime}(0)=0, x \in \Lambda,
\end{aligned}
$$

whose exact solution is given by $u(x)=x^{3}$.

If we apply the technique described in Section 4 with $N=3$, then the approximate solution can be written as

$$
u_{N}(x)=\sum_{i=0}^{3} c_{i} L_{i}^{(\alpha, \beta)}(x)=C^{T} \phi(x)
$$

and

$$
\mathbf{P}^{\left(\frac{5}{2}\right)}=\left(\begin{array}{cccc}
\Psi_{\frac{5}{2}}(0,0) & \Psi_{\frac{5}{2}}(0,1) & \Psi_{\frac{5}{2}}(0,2) & \Psi_{\frac{5}{2}}(0,3) \\
\Psi_{\frac{5}{2}}(1,0) & \Psi_{\frac{5}{2}}(1,1) & \Psi_{\frac{5}{2}}(1,2) & \Psi_{\frac{5}{2}}(1,3) \\
\Psi_{\frac{5}{2}}(2,0) & \Psi_{\frac{5}{2}}(2,1) & \Psi_{\frac{5}{2}}(2,2) & \Psi_{\frac{5}{2}}(2,3) \\
\Psi_{\frac{5}{2}}(3,0) & \Psi_{\frac{5}{2}}(3,1) & \Psi_{\frac{5}{2}}(3,2) & \Psi_{\frac{5}{2}}(3,3)
\end{array}\right), \quad G=\left(\begin{array}{l}
g_{0} \\
g_{1} \\
g_{2} \\
g_{3}
\end{array}\right)
$$

Using Eq. (28), we obtain

$$
\begin{aligned}
& 3 \Psi_{\frac{5}{2}}(0,2) c_{0}+3 \Psi_{\frac{5}{2}}(1,2) c_{1}+\left(1+3 \Psi_{\frac{5}{2}}(2,2)\right) c_{2}+3 \Psi_{\frac{5}{2}}(3,2) c_{3}+g_{2}=0 \\
& 3 \Psi_{\frac{5}{2}}(0,3) c_{0}+3 \Psi_{\frac{5}{2}}(1,3) c_{1}+3 \Psi_{\frac{5}{2}}(2,3) c_{2}+\left(1+3 \Psi_{\frac{5}{2}}(3,3)\right) c_{3}+g_{3}=0
\end{aligned}
$$


Table 2 The values of $c_{0}, c_{1}, c_{2}$ and $c_{3}$ for various choices of $\alpha$ and $\beta$ for Example 2

\begin{tabular}{|c|c|c|c|c|c|c|c|c|c|c|c|}
\hline $\bar{\alpha}$ & $\beta$ & $c_{0}$ & $c_{1}$ & $c_{2}$ & $\overline{c_{3}}$ & $\alpha$ & $\beta$ & $c_{0}$ & $c_{1}$ & $c_{2}$ & $c_{3}$ \\
\hline 0 & 1 & 6 & -18 & 18 & -6 & 0 & 2 & $\frac{3}{4}$ & $-\frac{9}{4}$ & $\frac{9}{4}$ & $-\frac{3}{4}$ \\
\hline 1 & & 24 & -36 & 24 & -6 & 1 & & 3 & $-\frac{9}{2}$ & 3 & $-\frac{3}{4}$ \\
\hline 2 & & 60 & -60 & 30 & -6 & 2 & & $\frac{15}{2}$ & $-\frac{15}{2}$ & $\frac{15}{4}$ & $-\frac{3}{4}$ \\
\hline 3 & & 120 & -90 & 36 & -6 & 3 & & 15 & $-\frac{45}{4}$ & $\frac{9}{2}$ & $-\frac{3}{4}$ \\
\hline 4 & & 210 & -126 & 42 & -6 & 4 & & $\frac{105}{4}$ & $-\frac{63}{4}$ & $\frac{21}{4}$ & $-\frac{3}{4}$ \\
\hline 0 & 3 & $\frac{2}{9}$ & $-\frac{2}{3}$ & $\frac{2}{3}$ & $-\frac{2}{9}$ & 0 & 4 & $\frac{3}{32}$ & $-\frac{9}{32}$ & $\frac{9}{32}$ & $-\frac{3}{32}$ \\
\hline 1 & & $\frac{8}{9}$ & $-\frac{4}{3}$ & $\frac{8}{9}$ & $-\frac{2}{9}$ & 1 & & $\frac{3}{8}$ & $-\frac{9}{16}$ & $\frac{3}{8}$ & $-\frac{3}{32}$ \\
\hline 2 & & $\frac{20}{9}$ & $-\frac{20}{9}$ & $\frac{10}{9}$ & $-\frac{2}{9}$ & 2 & & $\frac{15}{16}$ & $-\frac{15}{16}$ & $\frac{15}{32}$ & $-\frac{3}{32}$ \\
\hline 3 & & $\frac{40}{9}$ & $-\frac{30}{9}$ & $\frac{4}{3}$ & $-\frac{2}{9}$ & 3 & & $\frac{15}{8}$ & $-\frac{45}{32}$ & $\frac{9}{16}$ & $-\frac{3}{32}$ \\
\hline 4 & & $\frac{70}{9}$ & $-\frac{14}{3}$ & $\frac{14}{9}$ & $-\frac{2}{9}$ & 4 & & $\frac{105}{32}$ & $-\frac{63}{32}$ & $\frac{21}{32}$ & $-\frac{3}{32}$ \\
\hline
\end{tabular}

Now, applying Eq. (29), we get

$$
\begin{aligned}
& C^{T} \phi(0)=c_{0}+\frac{\Gamma(\alpha+2)}{\Gamma(\alpha+1)} c_{1}+\frac{\Gamma(\alpha+3)}{2 \Gamma(\alpha+1)} c_{2}+\frac{\Gamma(\alpha+4)}{6 \Gamma(\alpha+1)} c_{3}=0, \\
& C^{T} \mathbf{D}^{(1)} \phi(0)=-\beta c_{1}-\frac{\beta \Gamma(\alpha+3)}{\Gamma(\alpha+2)} c_{2}-\frac{\beta \Gamma(\alpha+4)}{2 \Gamma(\alpha+2)} c_{3}=0 .
\end{aligned}
$$

By solving the linear system (35)-(36), we get

$$
\begin{aligned}
& c_{0}=\frac{\alpha^{3}+6 \alpha^{2}+11 \alpha+6}{\beta^{3}}, \quad c_{1}=\frac{-3 \alpha^{2}-15 \alpha-18}{\beta^{3}}, \\
& c_{2}=\frac{6 \alpha+18}{\beta^{3}}, \quad c_{3}=\frac{-6}{\beta^{3}} .
\end{aligned}
$$

Thereby, we can write

$$
u_{N}(x)=\sum_{i=0}^{3} c_{i} L_{i}^{(\alpha, \beta)}(x)=x^{3} .
$$

Numerical results will not be presented since the exact solution is obtained. We have presented the values of the four unknown coefficients with various choices of $\alpha$ and $\beta$ in Table 2.

Example 3 Consider the following fractional initial value problem:

$$
D^{\frac{3}{2}} u(x)+3 u(x)=\gamma^{\frac{3}{2}} e^{\gamma x}+3 e^{\gamma x}, \quad u(0)=1, u^{\prime}(0)=\gamma, x \in(0,20),
$$

whose exact solution is given by $u(x)=e^{\gamma x}$.

Table 3 lists the maximum absolute error of $u-u_{N}$, using the operational matrix of fractional integration for the modified generalized Laguerre with tau method for $\gamma=0.01$, and various choices of $N, \alpha$ and $\beta$. From Table 3, we can achieve a good approximation to the exact solution by using a few terms of modified generalized Laguerre polynomials. Also, the maximum absolute error for $N=10$ and different values of $\gamma, \alpha$, and $\beta$ are shown in Table 4. 
Table 3 Maximum absolute error for $\gamma=0.01$ and different values of $\alpha, \beta$ and $N$ for Example 3

\begin{tabular}{lllllll}
\hline $\boldsymbol{N}$ & $\boldsymbol{\alpha}$ & $\boldsymbol{\beta}$ & Error & $\boldsymbol{\alpha}$ & $\boldsymbol{\beta}$ & Error \\
\hline 10 & 0 & 1 & $3.50 \cdot 10^{-3}$ & 1 & 2 & $9.88 \cdot 10^{-2}$ \\
20 & & & $3.67 \cdot 10^{-4}$ & & & $5.84 \cdot 10^{-2}$ \\
30 & & & 0 & & & $1.46 \cdot 10^{-2}$ \\
40 & & & 0 & & & $1.83 \cdot 10^{-3}$ \\
50 & & & 0 & & & 0 \\
10 & 3 & 2 & $7.95 \cdot 10^{-2}$ & 4 & 2 & $4.96 \cdot 10^{-2}$ \\
20 & & & $9.36 \cdot 10^{-3}$ & & & $3.27 \cdot 10^{-3}$ \\
30 & & & $3.17 \cdot 10^{-4}$ & & & $1.67 \cdot 10^{-9}$ \\
40 & & & $1.53 \cdot 10^{-14}$ & & & $4.13 \cdot 10^{-16}$ \\
50 & & & $1.54 \cdot 10^{-14}$ & & & $6.11 \cdot 10^{-16}$ \\
\hline
\end{tabular}

Table 4 Maximum absolute error for $N=10$ and different values of $\gamma, \alpha$, and $\beta$ for Example 3

\begin{tabular}{|c|c|c|c|}
\hline$\gamma$ & $\alpha \beta$ Error & $\alpha \beta$ Error & $\alpha \beta$ Error \\
\hline 0.2 & $012.52 \cdot 10^{-1}$ & $213.65 \cdot 10^{-2}$ & $119.65 \cdot 10^{-2}$ \\
\hline 0.1 & $9.73 \cdot 10^{-2}$ & $1.48 \cdot 10^{-2}$ & $3.84 \cdot 10^{-2}$ \\
\hline 0.01 & $3.51 \cdot 10^{-3}$ & $5.28 \cdot 10^{-4}$ & $1.42 \cdot 10^{-3}$ \\
\hline 0.02 & $9.70 \cdot 10^{-3}$ & $1.48 \cdot 10^{-3}$ & $3.92 \cdot 10^{-3}$ \\
\hline 0.05 & $3.56 \cdot 10^{-2}$ & $5.60 \cdot 10^{-3}$ & $1.46 \cdot 10^{-2}$ \\
\hline
\end{tabular}

\section{Conclusion}

In this paper, we introduced a general formulation for the modified generalized Laguerre operational matrix of fractional integration. Fractional integration is described in the Riemann-Liouville sense. We have presented an accurate direct solver for the general multi-term linear fractional-order differential equations with initial conditions on a semiinfinite domain by using modified generalized Laguerre tau approximation based on the modified generalized Laguerre operational matrix of fractional integration.

The numerical results given in the previous section demonstrate good accuracy of this algorithm. Moreover, the solutions obtained using the suggested algorithm show that this algorithm with a small number of modified generalized Laguerre polynomials gives a satisfactory result.

\section{Competing interests}

The authors declare that they have no competing interests.

Authors' contributions

The authors have equal contributions to each part of this article. All the authors read and approved the final manuscript.

\section{Acknowledgements}

This study was supported by the Deanship of Scientific Research of King Abdulaziz University. The authors would like to thank the editor and the reviewers for their constructive comments and suggestions to improve the quality of the article.

Received: 26 March 2012 Accepted: 21 September 2012 Published: 14 October 2012

\section{References}

1. Podlubny, I: Fractional Differential Equations. Academic Press, San Diego (1999)

2. Das, S: Functional Fractional Calculus for System Identification and Controls. Springer, New York (2008)

3. Kilbas, AA, Srivastava, HM, Trujillo, JJ: Theory and Applications of Fractional Differential Equations. Elsevier, San Diego (2006)

4. Baleanu, D, Diethelm, K, Scalas, E, Trujillo, JJ: Fractional Calculus Models and Numerical Methods. Series on Complexity, Nonlinearity and Chaos. World Scientific, Singapore (2012)

5. Chen, A, Chen, Y: Existence of solutions to nonlinear Langevin equation involving two fractional orders with boundary value conditions. Bound. Value Probl. 2011, 17 (2011)

6. Bhrawy, AH, Al-Shomrani, MM: A shifted Legendre spectral method for fractional-order multi-point boundary value problems. Adv. Differ. Equ. 2012, 8 (2012) 
7. Baleanu, D, Mustafa, OG, Agarwal, RP: An existence result for a superlinear fractional differential equation. Appl. Math. Lett. 23, 1129-1132 (2010)

8. Baleanu, D, Mustafa, OG, Agarwal, RP: On the solution set for a class of sequential fractional differential equations. J. Phys. A, Math. Theor. 43, 385209 (2010)

9. Lakestani, M, Dehghan, M, Irandoust-pakchin, S: The construction of operational matrix of fractional derivatives using B-spline functions. Commun. Nonlinear Sci. Numer. Simul. 17, 1149-1162 (2012)

10. Sabatier, J, Nguyen, H, Farges, C, Deletage, J-Y, Moreau, X, Guillemard, F, Bavoux, B: Fractional models for thermal modeling and temperature estimation of a transistor junction. Adv. Differ. Equ. 2011, 687363 (2011)

11. Pedas, A, Tamme, E: Piecewise polynomial collocation for linear boundary value problems of fractional differential equations. J. Comput. Appl. Math. 236, 3349-3359 (2012)

12. Yuzbasi, S: Numerical solution of the Bagley-Torvik equation by the Bessel collocation method. Math. Methods Appl. Sci. (2012). doi:10.1002/mma.2588

13. Ahmad, B, Nieto, JJ, Alsaedi, A, El-Shahed, M: A study of nonlinear Langevin equation involving two fractional orders in different intervals. Nonlinear Anal., Real World Appl. 13, 599-606 (2012)

14. Canuto, C, Hussaini, MY, Quarteroni, A, Zang, TA: Spectral Methods in Fluid Dynamics. Springer, New York (1989)

15. Boyd, JP: Chebyshev and Fourier Spectral Methods, 2nd edn. Dover, Mineola (2001)

16. Doha, EH, Bhrawy, AH, Saker, MA: On the derivatives of Bernstein polynomials: An application for the solution of higher even-order differential equations. Bound. Value Probl. 2011, 16 (2011)

17. Doha, EH, Abd-Elhameed, WM, Bhrawy, AH: Efficient spectral ultraspherical- Galerkin algorithms for the direct solution of 2nd-order linear differential equations. Appl. Math. Model. 33, 1982-1996 (2009)

18. Saadatmandi, A, Dehghan, M: A new operational matrix for solving fractional-order differential equations. Comput. Math. Appl. 59, 1326-1336 (2010)

19. Doha, EH, Bhrawy, AH, Ezz-Eldien, SS: Efficient Chebyshev spectral methods for solving multi-term fractional orders differential equations. Appl. Math. Model. 35, 5662-5672 (2011)

20. Bhrawy, AH, Alofi, AS, Ezz-Eldien, SS: A quadrature tau method for fractional differential equations with variable coefficients. Appl. Math. Lett. 24, 2146-2152 (2011)

21. Guo, B-Y, Zhang, X-Y: A new generalized Laguerre approximation and its applications. J. Comput. Appl. Math. 181, 342-363 (2005)

22. Guo, B-Y, Wang, L-L: Modified Laguerre pseudospectral method refined by multidomain Legendre pseudospectral approximation. J. Comput. Appl. Math. 190, 304-324 (2006)

23. Funaro, D: Estimates of Laguerre spectral projectors in Sobolev spaces. In: Brezinski, C, Gori, L, Ronveaux, A (eds.) Orthogonal Polynomials and Their Applications, pp. 263-266. Scientific Publishing Co., Singapore (1991)

24. Yan, J-P, Guo, B-Y: A collocation method for initial value problems of second order ODEs by using Laguerre function. Numer. Math. Theor. Meth. Appl. 4, 283-295 (2011)

25. Gulsu, M, Gurbuz, B, Ozturk, Y, Sezer, M: Laguerre polynomial approach for solving linear delay difference equations. Appl. Math. Comput. 217, 6765-6776 (2011)

26. Doha, EH, Bhrawy, AH, Ezz-Eldien, SS: A Chebyshev spectral method based on operational matrix for initial and boundary value problems of fractional order. Comput. Math. Appl. 62, 2364-2373 (2011)

27. Doha, EH, Bhrawy, AH, Ezz-Eldien, SS: A new Jacobi operational matrix: an application for solving fractional differentia equations. Appl. Math. Model. 36, 4931-4943 (2012)

28. Bhrawy, AH, Alghamdi, MA: A shifted Jacobi-Gauss-Lobatto Collocation method for solving nonlinear fractional Langevin equation. Bound. Value Probl. 2012, 62 (2012)

29. El-Danaf, TS, Ramadan, MA, Sherif, MN: Error analysis, stability, and numerical solutions of fractional-order differential equations. Int. J. Pure Appl. Math. 76, 647-659 (2012)

30. Kadem, A, Baleanu, D: Fractional radiative transfer equation within Chebyshev spectral approach. Comput. Math. Appl. 59, 1865-1873 (2010)

31. Jiang, Y-L, Ding, X-L: Waveform relaxation methods for fractional differential equations with the Caputo derivatives. J. Comput. Appl. Math. (2012). doi:10.1016/j.cam.2012.08.018

32. Bhrawy, AH, Tharwat, MM, Yildirim, A: A new formula for fractional integrals of Chebyshev polynomials: Application for solving multi-term fractional differential equations. Appl. Math. Model. (2012). doi:10.1016/j.apm.2012.08.022

33. Bhrawy, AH, Tharwat, MM, Alghamdi, MA: A new operational matrix of fractional integration for shifted Jacob polynomials. Bull. Malays. Math. Soc. (2012, accepted)

34. Paraskevopoulos, PN: Chebyshev series approach to system identification, analysis and control. J. Franklin Inst. 316, 135-157 (1983)

35. Doha, EH, Ahmed, HM, El-Soubhy, SI: Explicit formulae for the coefficients of integrated expansions of Laguerre and Hermite polynomials and their integrals. Integral Transforms Spec. Funct. 20, 491-503 (2009)

36. Singh, AK, Singh, VK, Singh, OP: The Bernstein operational matrix of integration. Appl. Math. Sci. 3(49), 2427-2436 (2009)

37. Bhrawy, AH, Alofi, AS: The operational matrix of fractional integration for shifted Chebyshev polynomials. Appl. Math. Lett. 26, 25-31 (2013)

38. Ford, NJ, Connolly, JA: Systems-based decomposition schemes for the approximate solution of multi-term fractional differential equations. J. Comput. Appl. Math. 229, 382-391 (2009)

doi:10.1186/1687-1847-2012-179

Cite this article as: Bhrawy et al.: A new modified generalized Laguerre operational matrix of fractional integration for solving fractional differential equations on the half line. Advances in Difference Equations 2012 0:179. 